\title{
The Impact of Pain and Other Symptoms on Quality of Life in Women With Relapsing-Remitting Multiple Sclerosis
}

\author{
Pamela K. Newland, PhD, RN [Assistant professor], \\ School of Nursing, Southern Illinois University Edwardsville, Edwardsville, IL \\ Robert T. Naismith, MD [Assistant professor], and \\ Department of Neurology, Washington University School of Medicine, St. Louis, MO \\ Margaret Ullione, PhD, RN [Director of the education] \\ Research and Center for Practice Excellence, Barnes Jewish Hospital, St. Louis, MO \\ Pamela K. Newland: pnewlan@siue.edu
}

\section{Abstract}

The purpose of this study was to assess pain, fatigue, depression, sleep disturbance, and quality of life (QOL) in women with relapsing-remitting multiple sclerosis (RRMS) compared with healthy controls. A prospective, cross-sectional, matched-control study was conducted in women with RRMS compared with healthy women. Compared with healthy women, women with RRMS had (a) greater pain presence over 7 days (67\%), (b) higher pain intensity, and (c) more pain interference. Pain had a negative impact on fatigue, depression, and sleep in both groups. In all participants, fatigue, depression, and sleep disturbance contributed to decreased mental QOL (mental component summary of QOL scores). Pain has significant nursing implications for women with RRMS. Pain often occurs in association with fatigue, depression, and sleep disturbance, which can lead to a decreased mental QOL.

\begin{abstract}
Multiple sclerosis (MS) is a chronic condition that causes a wide variety of neurological signs, symptoms, and deficits. The hallmark of MS is the demyelination of neurons, which consists of a loss of myelin, relative preservation of axons, and the formation of gliosis (astrocytic scars) on the myelin sheath of the central nervous system (CNS; Paty \& Arnold, 2002; Wingerchuk, Lucchinetti, \& Noseworthy, 2001). There are often objective and visible physical deficits in ambulation, vision, and arm coordination; other objective findings include bowel and bladder function, paresthesias, speech, and swallowing (Hauser \& Goodkin, 2005; Svendsen et al., 2003).

The less visible, subjective symptoms of pain, fatigue, and depression are common and burdensome and adversely affect quality of life (Bakshi et al., 2000; Svendsen, Jensen, Hansen, \& Bach, 2005; Svendsen et al., 2003). In particular, pain has been documented as a significant symptom experienced by persons with MS (Beiske, Pedersen, Czujko, \& Myhr, 2004; Svendsen et al., 2005), and it remains undertreated by healthcare providers (Ehde, Osborne, Hanley, Jensen, \& Kraft, 2006). Fatigue has a substantial impact on quality of life in persons with MS by reducing physical stamina, interfering with the performance of responsibilities at home and work, and limiting social interactions (Mohr, Hart, \& Goldberg, 2003). Depression is another symptom frequently experienced by persons with MS (Chwastiak \& Ehde, 2007; Newland, Wipke-Tevis, Williams, Rantz, \& Petroski, 2005) and continues to be undertreated
\end{abstract}


(Chwastiak \& Ehde, 2007). In addition, sleep disturbance, such as insomnia, hypersomnia, and hyposomnia, has recently been recognized in persons with MS (Fleming \& Pollak, 2005).

\section{Background}

Relapsing-remitting multiple sclerosis (RRMS) is the most common MS subtype (Ehde et al, 2003; Kalia \& O'Connor, 2005), with women constituting most of the affected individuals. The incidence of pain related to MS ranges from 53\% to 86\% (Kalia \& O'Connor, 2005; Newland et al., 2005; Svendsen et al., 2003). Pain with or without other symptoms, such as fatigue, depression, and sleep disturbance, deserves further study to determine its impact on quality of life, especially because some symptoms are under-treated. The purpose of this study was to examine the differences in pain, fatigue, depression, and sleep disturbance and their impact on quality of life between women with RRMS and healthy women.

\section{Method}

Forty women with RRMS were recruited from a university MS center during their regularly scheduled appointments. Participants were older than 18 years and had been diagnosed with MS for at least 6 months. Patients excluded were those with another autoimmune or neurological disease, cancer, diagnosis of a major psychiatric disorder, or any other chronic illness that may cause pain (e.g., diabetes mellitus, chronic renal failure, or osteoarthritis).

Forty healthy women matched by age ( \pm 2 years) and living location (urban and rural) were recruited from a Midwest metropolitan community via posted flyers at a local hospital and health center. For this study, healthy women were defined by self-report with the same exclusions as the MS sample. Approval for the study was obtained from the University of Missouri-Columbia and Washington University School of Medicine. Before the start of the study, participants read study information and gave informed consent.

\section{Measures}

Demographic variables, such as marital status, employment status, educational level, and type and dose of pain medication, and other information were used in this study, not the effectiveness of medications. Pain was measured with two instruments: the Brief Pain Inventory-Long Form (BPI-LF) and the McGill Pain Questionnaire Short Form (MPQ-SF). The BPI-LF (Daut, Cleeland, \& Flannery, 1983) measured the presence, average intensity, location, and how pain interferes with daily life over the last 7 days, categorizing pain as "yes" for pain and "no" for no pain. The BPI-LF consists of numeric rating scale items ranging from 0 (no pain) to 10 (pain as bad as you can imagine) to measure the intensity of pain over the last 7 days and how pain interferes with daily life (e.g., general activity, mood, and walking). In addition, back and front views of a human figure diagram allow the participant to mark areas for pain location. Concurrent validity has been established previously in the MS literature (Ehde et al., 2003).

The MPQ-SF (Melzack, 1987) measured present pain intensity (PPI), present pain descriptors, and present pattern of pain. The MPQ-SF has 11 items to assess sensory dimensions of pain and 4 items for affective dimensions to describe present pain. Scoring for the intensity of each descriptor is through the use of a Likert scale categorized, with $0=$ none, $1=$ mild, $2=$ moderate, and $3=$ severe for each of the 15 descriptor words. A visual analogue scale and PPI also are included from the MPQ-SF to assess PPI. Scoring of the visual analogue scale is a continuous $0-$ to $10-\mathrm{cm}$ line with the anchors no pain to worst possible pain, and scoring of the PPI is 0 for no pain and 5 for excruciating pain. Concurrent validity has been established in MS participants (Svendsen et al., 2003, 2005). 
The Lee Fatigue Scale (Lee, Hicks, \& Nino-Murcia, 1991) was used to measure intensity of fatigue. Each of the 18 items is rated on a Likert scale from 0 (not at all fatigued) to 10 (extremely fatigued), with a possible score range of 0 to 180. Higher scores indicate a higher level of fatigue. Reliability has been established at .96 (Miaskowski \& Lee, 1999).

Depression intensity was measured using the Beck Depression Inventory (BDI). The BDI consists of 21 items rated on a 4-point scale and has a possible score range of 0 to 63 , with a higher score indicating more depression (scores above 29 indicate severe depression). The BDI has good reliability ( $\alpha=.81$ for nonclinical samples) and validity (Beck, Ward, Medelson, Mock, \& Erbaugh, 1961).

Sleep disturbance intensity was measured with the General Sleep Disturbance Scale (Lee, 1992), a 21-item 7-point scale. The scale yields a total score ranging between 0 and 147, with higher scores indicating greater sleep disturbance. Overall reliability is .88 , with alpha coefficients ranging from .62 to .82 .

Quality of life was measured with the Medical Outcomes Study Form 36 (SF-36; Ware \& Sherbourne, 1992). The SF-36 consists of 36 items in eight subscales with components of mental and physical health. Composite scores on the mental and physical component summary scores may range from 0 to 100 , with higher scores indicating better health-related quality of life. Validity and reliability of the SF-36 have been well established in participants with MS (Pittock et al., 2004; Svendsen et al., 2005).

Data were analyzed using the Statistical Analysis System 9.1 (SAS Institute Inc., Cary, NC). For demographics and outcomes that were categorical, either the chi-square test for homogeneity of proportions or Fisher's exact test was used. For ordinal data, Wilcoxon rank sum test was used. Frequencies were used for describing pain location over the last 7 days by calculating areas indicated on the body diagram (BPI-LF). Patterns of pain and pain descriptors were measured using frequencies of the words chosen (MPQ-SF). Two-way analysis of variance examined the effect of pain over the last 7 days on fatigue, depression, and sleep disturbance in both groups. Pearson correlation coefficients described the relationship of quality of life to pain, fatigue, depression, and sleep disturbance. Variables with a relatively strong $(r=.6)$ and significant correlation were then included in a stepwise regression model to determine the effect of each variable separately on mental and physical quality of life.

\section{Results}

\section{Sample Description}

The average age of the women with RRMS and healthy women was 44 years. Both groups of women were primarily Caucasian, married, well educated, and urban dwelling. Healthy women had more years of education, and a greater percentage was employed compared with women with RRMS (Table 1).

Study variables included pain, fatigue, depression, and sleep disturbance. The results for these measures are presented in Table 2. Pain was present twice as often in women with RRMS compared with healthy women ( $67 \%$ vs. $33 \%, p=.005)$. Pain intensity over 7 days was over twice as high for women with RRMS compared with healthy women (median $=5.0 \mathrm{vs.} 2.0$, $p=.02$ ). PPI was $50 \%$ higher for women with RRMS compared with healthy women (median $=3.0$ vs. $2.0, p=.03$ ). With regard to average pain intensity, women with RRMS reported higher average pain intensity compared with healthy women $(p=.001$; Figure 1$)$. Women with RRMS reported that pain interfered with general activities more than healthy women $(p=$. 0008; Figure 2). Fatigue $(p=.06)$ and depression intensity $(p=.14)$ were not significantly 
different between women with RRMS and healthy women $(p=.06)$. In contrast, sleep disturbance intensity was different in women with RRMS and healthy women $(p=.02)$.

Simultaneous correlations were conducted with regression analysis. A negative correlation indicated a diminished mental quality of life and fatigue, depression, and sleep disturbance intensity in all women (Table 3). No correlation was found between mental quality of life and pain intensity (average pain over the last 7 days) in all women. Likewise, a negative correlation indicated a diminished physical quality of life and fatigue in all women. Also, for physical quality of life, a negative correlation accounting for $20 \%$ of the variance was found for average pain intensity over the last 7 days (Table 3 ). Because a small portion of all women in this study reported having pain $(n=44)$ over the last 7 days, pain was not included as a predictor variable in the regression analysis.

A regression model of quality of life in both groups was created using predictors of fatigue, depression, and sleep disturbance by regression analyses with a forward selection procedure. The symptom of depression was entered first, and it accounted for the largest amount of variance of the regression model. Next, fatigue and sleep disturbance each increased the predictive ability of the regression model for mental quality of life as they were entered sequentially. For both groups, an increase in all three symptoms (depression, fatigue, and sleep disturbance intensity) was a predictor for decreased mental quality of life. Stepwise regression revealed that increased fatigue intensity was a predictor for decreased physical quality of life in all women, regardless of group.

Additional analysis was conducted on symptoms for women with RRMS and healthy women with and without pain. Fatigue intensity scores were high in both groups of women with and without pain $(p=.03)$. Depression intensity scores were higher in women with RRMS with pain compared with those without pain $(p=.002)$. Conversely, the depression intensity scores were similar in healthy women with pain and without pain $(p=.05)$. Sleep disturbance intensity scores also were higher for women with RRMS with pain than those of women without pain $(p=.03)$. Likewise, sleep disturbance intensity scores were high in both groups of women with pain and without pain $(p=.04)$.

\section{Discussion}

Our study provides important knowledge on how pain, fatigue, depression, and sleep disturbance affect women with RRMS. The use of a matched healthy group revealed that although the intensity of symptoms may differ, the presence of these symptoms is obviously not unique to RRMS. The higher prevalence of pain in women with RRMS compared with healthy women is similar to other findings (Archibald et al., 1994; Beiske et al., 2004; Moulin, 1998; Newland et al., 2005) in the population with MS. One explanation for the higher prevalence of pain in the women with RRMS may be due to the pathophysiology of MS. Injury as a result of demyelination in MS can lead to ectopic excitation at various areas of the spinal cord (Moulin, 1998; Woolf \& Max, 2001). The continuous exposure of the myelin sheath of the CNS can lead to a heightened sensation of pain (Ge et al., 2000).

The increase in present and average pain intensity in women with RRMS may be due to the exposure of the myelin sheath of the CNS to noxious stimuli (Morin, Bushnell, Luskin, \& Craig, 2002). Alternatively, the higher pain intensity may be due to the inflammatory processes of active demyelinated lesions in persons with RRMS (Moulin, 1998). Although the status of acute relapse and demyelination in the women with RRMS was not examined in this study, increased pain in women with RRMS may be attributed to an underlying inflammatory process or demyelination of the brain and spinal cord (Hauser \& Goodkin, 2005, Solaro et al., 2004; Wingerchuk, Lucchinetti, \& Noseworthy, 2001). More women with RRMS reported that pain 
interfered with their daily lives (Figure 1). Therefore, another important dimension that needs further monitoring is pain interference in women with RRMS.

The impact of pain on fatigue is complex. Because pain and fatigue are thought to share the same nerve pathway in the brain and spinal cord (Merkelbach, Sittinger, \& Koenig, 2002), pain may cause an increase in fatigue intensity. In addition, pain activates endocrine and metabolic mechanisms that are associated with fatigue and the stress response (Attarian, Brown, Duntley, Carter, \& Cross, 2004; Beiske et al., 2004). Pain and fatigue may share the same CNS pathways, thereby increasing catecholamine and cortisol levels. In response, the sympathetic nervous system (SNS) is on overload, leading to a vicious cycle of more fatigue (Reyes-Gibby, Mendoza, Wang, Anderson, \& Cleeland, 2003).

Pain and depression may be processed in the somatosensory pathways of the brain and spinal cord (Delgado, 2004). These areas are often affected with atrophy and demyelination in MS. In addition, pain and depression are processed in the limbic system of the cerebral cortex, which also modulates pain and manifests the affective-motivational dimension of pain (Bair, Robinson, Katon, \& Kroenke, 2003; Mohr et al., 2003). Not only does pain lead to depression, but depression plays a role in pain management (Bair et al., 2003).

Increases in sleep disturbance intensity in women with RRMS could be explained by the pathophysiology of RRMS. For example, demyelinated lesions and inflammatory responses from RRMS may disrupt the sleep-wake cycle by interfering with response of the suprachiasmatic nucleus of the brain (Attarian et al., 2004; Fleming \& Pollak, 2005). In addition, women with RRMS reported more daytime sleepiness, poor sleep quality, and hypnotic medication use than did healthy women.

In this study, the impact of pain on sleep disturbance intensity in all women could be explained by the physiological response of pain on the sleep-wake cycle. It has been hypothesized that the modulation of pain and regulation of the sleep-wake cycle share neurobiological pathways (Kundermann, Krieg, Schreiber, \& Lautenbacher, 2004). These data support previous research (Call-Schmidt \& Richardson, 2003) showing that pain acts as a stressor that can interfere with cortisol and other hormones that affect sleep.

The adverse impact of depression, fatigue, and sleep disturbance on mental quality of life is significant. Women with this set of symptoms may experience a negative psychological reaction, particularly with more restricted work and social relationships, and this disruption thereby decreases their mental quality of life. These findings concur with those of others (McCabe \& McKern, 2002; Miller \& Dishon, 2006; Pittock et al., 2004) that suggest that physical quality of life decreases in women with RRMS compared with healthy women. Moreover, the aspects of the decreased physical quality of life that accompany MS may lead to frequent physician visits, early retirement, loss of income, and social isolation (Pittock et al., 2004).

\section{Limitations}

The cross-sectional design limits to one time point the measurement of this set of symptoms on quality of life. Although these data do not support the exclusive use of a multidimensional pain instrument, comprehensive assessment of pain is needed in women with RRMS.

Surprisingly, pain had a relatively modest relationship to mental and physical quality of life in women. However, only a small subset of the women reported pain, so the correlations may not have been statistically stable enough to uncover more significant findings. It is possible that controlling for type of medications could strengthen future studies. 


\section{Nursing Implications and Future Research}

These findings lead to important information for nursing practice. Nurses, in all settings, should be aware of how best to assess for pain and other symptoms in women with RRMS and healthy women. Specifically, the use of the short version of the BPI (Cleeland \& Ryan, 1994) in the community clinical setting provides a succinct, valid, and reliable assessment of pain by nurses. These findings underscore the importance of carefully evaluating subjective perceptions of symptoms in all settings, with regard to the impact on the daily lives of women. Given that fatigue had the greatest adverse impact on physical quality of life in all women, attention needs to be concentrated on interventions to relieve fatigue. Strategies include encouraging rest between activities and exercise. Even more emphasis should be placed on early identification of depression to improve mental quality of life. Strategies nurses can employ include screening for depression with a standardized tool at each encounter. Nurses can discuss ways to address depression and provide appropriate referrals to health specialists.

Additional research is needed to develop interventions for pain to reduce fatigue, depression, and sleep disturbance. Future intervention studies are necessary to examine women with RRMS to determine more positive health outcomes.

\section{Summary}

Overall, these data indicate that women with RRMS represent a unique population who experience pain differently than do healthy women. Furthermore, the findings from this study are an important step in providing a better understanding of the symptoms that impact quality life in women. All women should be assessed routinely for pain, fatigue, depression, and sleep disturbance. Effective pain management may decrease the intensity of fatigue, depression, and sleep disturbance in all women. In addition, in women with multiple symptoms, treatment of depression may improve mental quality of life, whereas treatment of fatigue may improve physical quality of life.

\section{Acknowledgments}

This research was supported by a grant from Omicron Psi Sigma Theta Tau International (Pamela Newland) and National Institutes of Health K23NS052430-01A1 (Robert Naismith). The authors thank Anne Cross, MD, for her thoughtful review of this article.

\section{References}

Archibald CJ, McGrath PJ, Ritvo PG, Fisk JD, Bhan V, Maxner CE, et al. Pain prevalence, severity, and impact in a clinic sample of multiple sclerosis patients. Pain 1994;58(1):89-93. [PubMed: 7970843]

Attarian HP, Brown KM, Duntley SP, Carter JD, Cross AH. The relationship of sleep disturbances and fatigue in multiple sclerosis. Archives of Neurology 2004;61(4):525-528. [PubMed: 15096400]

Bakshi R, Shikh ZA, Miletich RS, Czarnecki D, Dmorchowski J, Henschel K. Fatigue in multiple sclerosis and its relationship to depression and neurologic disability. Multiple Sclerosis 2000;6(3):181-185. [PubMed: 10871830]

Bair MJ, Robinson RI, Katon W, Kroenke K. Depression and pain comorbidity: A literature review. Archives of Internal Medicine 2003;163(20):2433-2445. [PubMed: 14609780]

Beck AT, Ward CH, Medelson M, Mock J, Erbaugh J. An inventory for measuring depression. Archives of General Psychiatry 1961;4:561-571. [PubMed: 13688369]

Beiske AG, Pedersen ED, Czujko B, Myhr KM. Pain and sensory complaints in multiple sclerosis. European Journal of Neurology 2004;11(7):479-482. [PubMed: 15257687]

Call-Schmidt TA, Richardson SJ. Prevalence of sleep disturbance and its relationship to pain in adults with chronic pain. Pain Management Nursing 2003;4(3):124-133. [PubMed: 14566710] 
Chwastiak LA, Ehde DM. Psychiatric issues in multiple sclerosis. Psychiatric Clinics of North America 2007;30(4):803-817. [PubMed: 17938046]

Cleeland CS, Ryan KM. Pain assessment: Global use of the Brief Pain Inventory. Annals of the Academy of Medicine, Singapore 1994;23(2):129-138.

Daut RL, Cleeland CS, Flannery RC. Development of the Wisconsin Brief Pain Questionnaire to assess pain in cancer and other disease. Pain 1983;17(2):197-210. [PubMed: 6646795]

Delgado PL. Common pathways of depression and pain. Journal of Clinical Psychiatry 2004;65(Suppl 12):16-19. [PubMed: 15315473]

Ehde DM, Gibbons LE, Chwastiak L, Bombardier CH, Sullivan MD, Kraft GH. Chronic pain in a large community sample of persons with multiple sclerosis. Multiple Sclerosis 2003;9(6):605-611. [PubMed: 14664474]

Ehde DM, Osborne TL, Hanley MA, Jensen MP, Kraft GH. The scope and nature of pain in persons with multiple sclerosis. Multiple Sclerosis 2006;12(5):629-638. [PubMed: 17086910]

Fleming WE, Pollak CP. Sleep disorders in multiple sclerosis. Seminars in Neurology 2005;25(1):6468. [PubMed: 15798938]

Ge Y, Grossman RJ, Udupa JK, Wei L, Mannon LJ, Polansky M, et al. Brain atrophy in relapsingremitting multiple sclerosis and secondary progressive multiple sclerosis: Longitudinal quantitative analysis. Radiology 2000;214(3):665-670. [PubMed: 10715027]

Hauser, SL.; Goodkin, DS. Multiple sclerosis and other demyelinating diseases. In: Hauser, S., editor. Harrison's principles of internal medicine. 16. New York: McGraw-Hill; 2005. p. 2461-2472.

Kalia LV, O'Connor PW. Severity of chronic pain and its relationship to quality of life in multiple sclerosis. Multiple Sclerosis 2005;11(3):322-327. [PubMed: 15957515]

Kundermann B, Krieg JC, Schreiber W, Lautenbacher S. The effect of sleep deprivation on pain. Pain Research and Management 2004;9(1):25-32. [PubMed: 15007400]

Lee KA. Self-reported sleep disturbances in employed women. Sleep 1992;15(6):493-498. [PubMed: 1475563]

Lee KA, Hicks G, Nino-Murcia G. Validity and reliability of a scale to assess fatigue. Psychiatric Research 1991;36(3):291-298.

McCabe MP, McKern S. Quality of life and multiple sclerosis: Comparison between people with multiple sclerosis and people from the general population. Journal of Clinical Psychology in Medical Settings 2002;9(4):287-295.

Melzack R. The short-form McGill Pain Questionnaire. Pain 1987;30(2):191-197. [PubMed: 3670870]

Merkelbach S, Sittinger H, Koenig J. Is there a differential impact of fatigue and physical disability on quality of life in multiple sclerosis? Journal of Nervous and Mental Disease 2002;190(6):388-393. [PubMed: 12080209]

Miaskowski C, Lee K. Pain, fatigue, and sleep disturbances in oncology outpatients receiving radiation therapy for bone metastasis: A pilot study. Journal of Pain and Symptom Management 1999;17:320 332. [PubMed: 10355211]

Miller A, Dishon S. Health-related quality of life in multiple sclerosis: The impact of disability, gender and employment status. Quality of Life Research 2006;15(2):259-271. [PubMed: 16468081]

Mohr D, Hart SL, Goldberg A. Effects of treatment for depression on fatigue in multiple sclerosis. Psychosomatic Medicine 2003;65(4):542-547. [PubMed: 12883103]

Morin C, Bushnell MC, Luskin MB, Craig AD. Disruption of thermal perception in a multiple sclerosis patient with central pain. Clinical Journal of Pain 2002;18(3):191-195. [PubMed: 12048421]

Moulin DE. Pain in central and peripheral demyelinating disorders. Neurology Clinics 1998;16(4):889_ 897.

Newland PK, Wipke-Tevis DD, Williams DA, Rantz MJ, Petroski GF. Impact of pain on outcomes in long-term care residents with and without multiple sclerosis. Journal of the American Geriatrics Society 2005;53(9):1490-1496. [PubMed: 16137277]

Paty D, Arnold D. The lesions of multiple sclerosis. New England Journal of Medicine 2002;346(6):199_ 200. [PubMed: 11796856] 
Pittock SJ, Mayr WT, McClelland RL, Jørgensen NW, Weigand S, Noseworthy JH, et al. Quality of life is favorable for most patients with multiple sclerosis. Archives of Neurology 2004;61(5):679-686. [PubMed: 15148144]

Reyes-Gibby CC, Mendoza TR, Wang S, Anderson KO, Cleeland CS. Pain and fatigue in communitydwelling adults. Pain Medicine 2003;4(3):231-237. [PubMed: 12974822]

Solaro C, Brichetto G, Amato MP, Cocco E, Columbo B, D'Aleo G, et al. The prevalence of pain in multiple sclerosis. Neurology 2004;63(5):919-921. [PubMed: 15365151]

Svendsen KB, Jensen TS, Hansen HJ, Bach FW. Sensory function and quality of life in patients with multiple sclerosis and pain. Pain 2005;114(3):473-481. [PubMed: 15777872]

Svendsen KB, Jensen TS, Overvad K, Hansen HJ, Kock-Henriksen N, Bach FW. Pain in patients with multiple sclerosis. Archives of Neurology 2003;60(8):1089-1094. [PubMed: 12925364]

Ware JE, Sherbourne CD. The MOS 36-Item Short-Form Health Survey (SF-36): Conceptual framework and item selection. Medical Care 1992;30(6):473-483. [PubMed: 1593914]

Wingerchuk DM, Lucchinetti CF, Noseworthy JH. Multiple sclerosis: Current pathophysiological concepts. Laboratory Investigation 2001;81(3):263-281. [PubMed: 11310820]

Woolf CJ, Max MB. Mechanism-based pain diagnosis issues for analgesic drug development. Anesthesiology 2001;95:241-249. [PubMed: 11465563] 


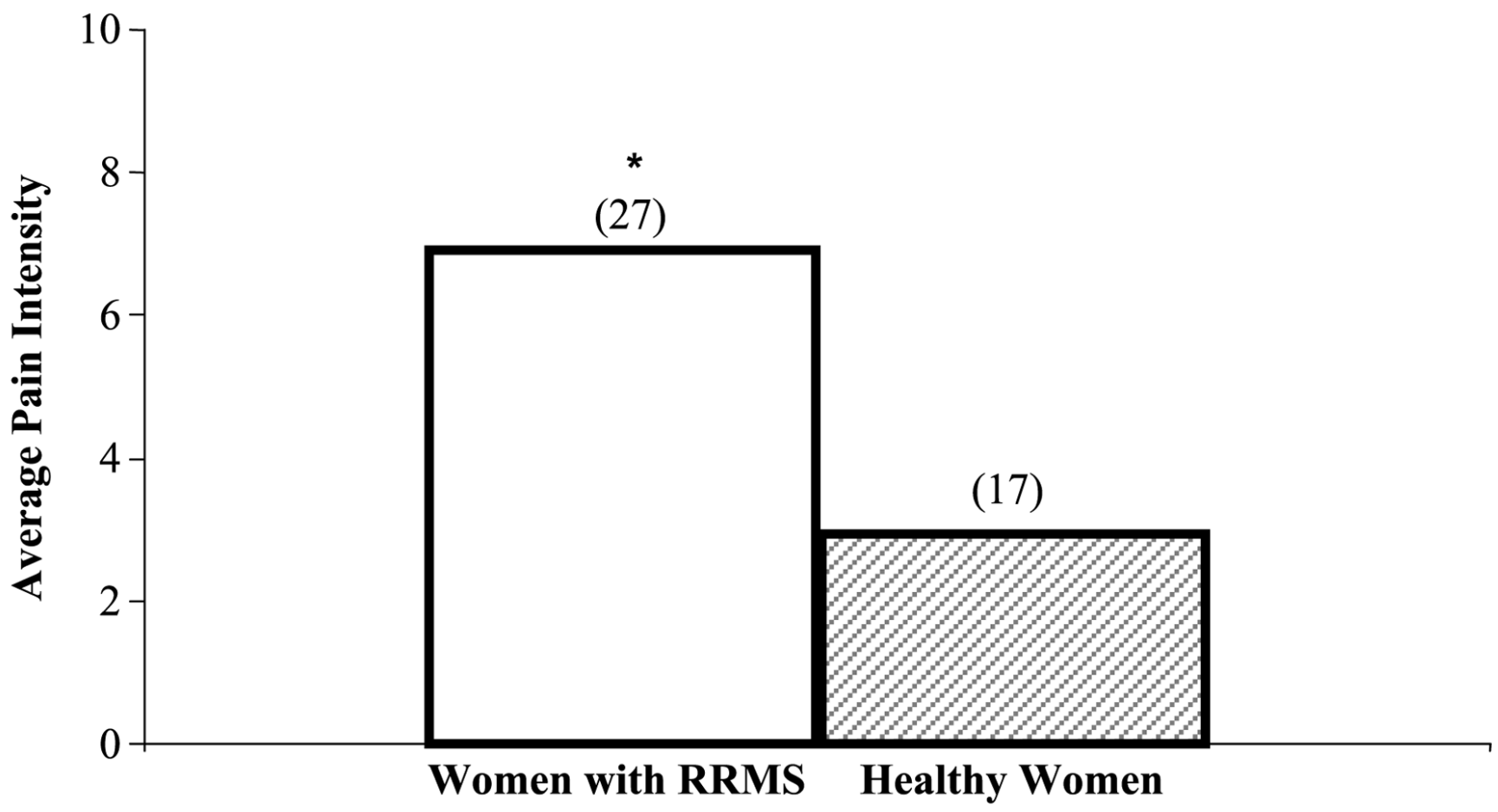

FIGURE 1.

Median Average Pain Intensity Over the Last 7 Days in Women With RRMS and Healthy Women

Note. Average pain intensity was measured using the Brief Pain Inventory-Long Form, ${ }^{*} p=$. $001(n)$, women with RRMS > healthy women. RRMS = relapsing-remitting multiple sclerosis. 


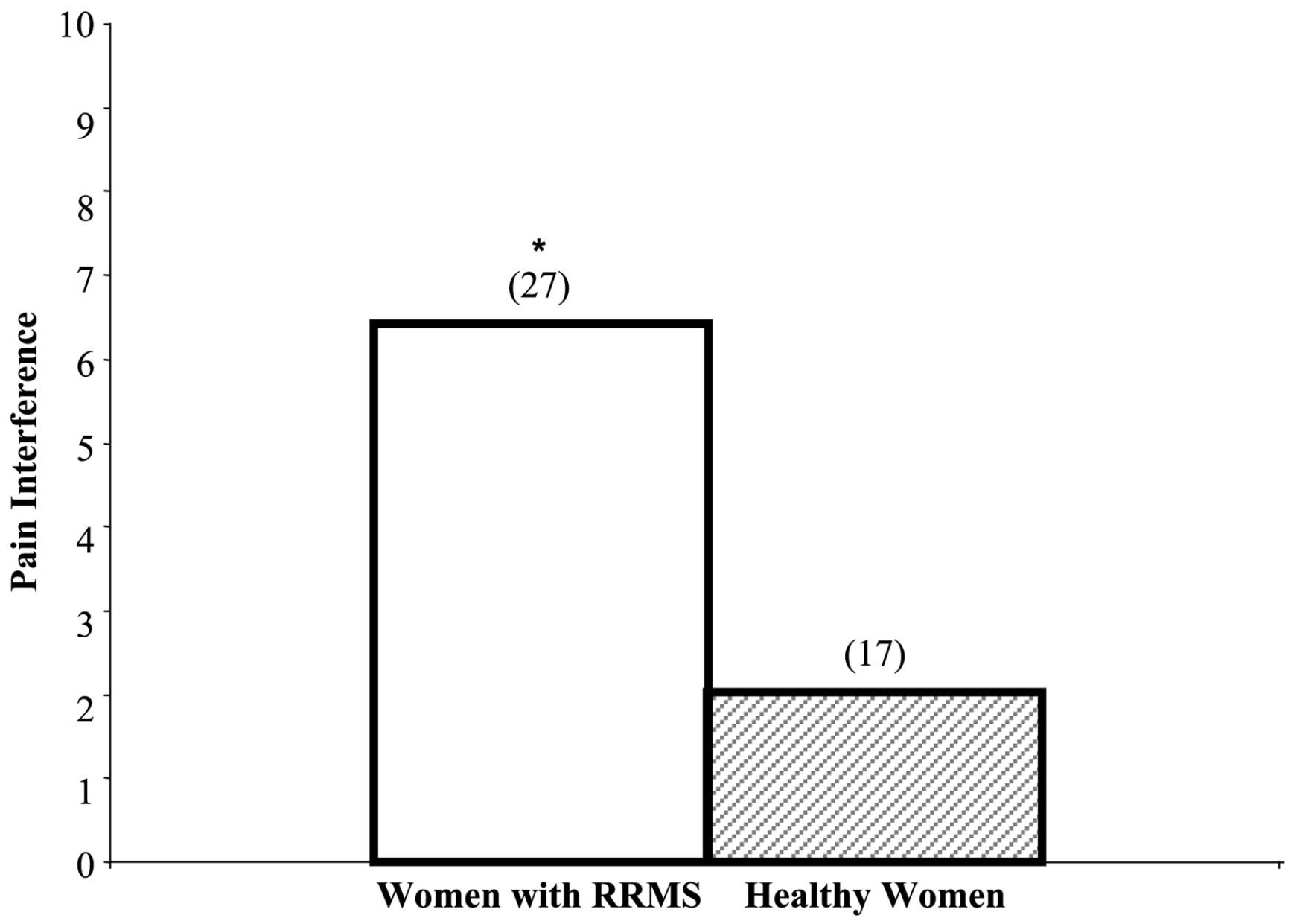

FIGURE 2.

Median Pain Interference Over the Last 7 Days in Women With RRMS and Healthy Women Note. Pain interference was measured using the Brief Pain Inventory-Long Form, $* p=.0008$ $(n)$, women with RRMS > healthy women. Pain interference is defined as interference with general activities. RRMS = relapsing-remitting multiple sclerosis. 
TABLE 1

Sample Demographics

\begin{tabular}{|c|c|c|c|}
\hline Variables & Women With RRMS $(n=40)$ & Healthy Women $(n=40)$ & Overall Sample $(n=80)$ \\
\hline Age (years), $M(S D)$ & $43.8(9.2)$ & $43.4(10.3)$ & $43.6(3.6)$ \\
\hline Range & $26-69$ & $24-69$ & $24-69$ \\
\hline Time since diagnosis (years), $M(S D)$ & $8.7(7.3)$ & N/A & N/A \\
\hline Education (years), $M(S D)$ & $14.3(2.7)^{*}$ & $16.5(2.9)$ & $15.1(2.9)$ \\
\hline PASAT, $M(S D)$ & $36.2(11.7)^{* *}$ & $52.3(4.6)$ & N/A \\
\hline \multicolumn{4}{|l|}{ Marital status (\%) } \\
\hline Single & 20 & 22.5 & 21.3 \\
\hline Married & 55 & 72.5 & 64.1 \\
\hline Divorced or widowed & 20 & 5 & 12.5 \\
\hline Separated & 5 & 0 & 2.5 \\
\hline \multicolumn{4}{|l|}{ Race $(\%)$} \\
\hline White & 77.5 & 85 & 81.2 \\
\hline African American ${ }^{a}$ & 22.5 & 12.5 & 17.5 \\
\hline Hispanic & 0 & 2.5 & 1.3 \\
\hline Asian or Pacific Islander & 0 & 0 & 0 \\
\hline Native American & 0 & 0 & 0 \\
\hline Other & 0 & 0 & 0 \\
\hline \multicolumn{4}{|l|}{ Living location (\%) } \\
\hline Urban & 77.5 & 77.5 & 77.5 \\
\hline Rural & 22.5 & 22.5 & 22.5 \\
\hline \multicolumn{4}{|l|}{ Living arrangement (\%) } \\
\hline Alone & 15 & 15 & 15 \\
\hline With spouse & 57.5 & 70 & 63.8 \\
\hline Family, friends & 27.5 & 15 & 21.2 \\
\hline Institution & 0 & 0 & 0 \\
\hline \multicolumn{4}{|l|}{ Employment (\%) } \\
\hline Employed & $37.5^{* *}$ & 75 & 56.2 \\
\hline Unemployed & 25 & 15 & 20 \\
\hline Disabled & 32.5 & 0 & 16.3 \\
\hline Other & 5 & 10 & 7.5 \\
\hline
\end{tabular}

Note. Percentages are based on the total for each group and may not total 100 due to rounding. RRMS = relapsing-remitting multiple sclerosis; $S D=$ standard deviation; PASAT $=$ Paced Auditory Serial Addition Test; N/A = not applicable.

${ }^{a}$ The African American sample reflects racial diversity of the area.

$*$ p $=.001$.

$* *$

$p<.0001$. 


\section{TABLE 2}

Symptoms and Quality of Life Variables in Women With RRMS and Healthy Women

\begin{tabular}{|lcc|}
\hline Variable & Women With RRMS & Healthy Women \\
Pain & $6.0(2.45)$ & $3.7(1.23)$ \\
Fatigue & $4.1(2.26)$ & $2.0(2.31)$ \\
Depression & $10.1(8.56)$ & $7.0(6.15)$ \\
Sleep disturbance & $26.7(11.04)$ & $21.1(10.81)$ \\
Mental quality of life & $42.72(14.85)$ & $48.31(9.82)$ \\
Physical quality of life & $38.03(10.01)$ & $52.0(8.5)$ \\
\hline
\end{tabular}

Note. Values are presented as $M(S D)$. RRMS = relapsing-remitting multiple sclerosis. 


\section{TABLE 3}

Correlations of HRQOL and Symptom Intensity Variables for Women With RRMS and Healthy Women $(n=$ $80)^{a}$

\begin{tabular}{|lllll|}
\hline HRQOL Component Variable & Pain Over the Last 7 Days & Fatigue & Depression & Sleep Disturbance \\
Mental & $-.25, p=.09$ & $-.63, p<.0001$ & $-.69, p<.0001$ & $-.57, p<.0001$ \\
Physical & $-.45, p=.002$ & $-.58, p<.0001$ & $-.43, p<.0001$ & $-.45, p<.0001$ \\
\hline
\end{tabular}

Note . Mental = mental component of the SF-36; physical = physical component of the SF-36; HRQOL = health-related quality of life; RRMS = relapsing-remitting multiple sclerosis; SF-36 = Short Form 36.

$a_{n=44 \text { for pain correlations for all women. }}$ 